\title{
Prediction of local wind climatology from Met Office models: Virtual Met Mast techniques
}

Article

Accepted Version

Standen, J., Wilson, C., Vosper, S. and Clark, P. (2017)

Prediction of local wind climatology from Met Office models: Virtual Met Mast techniques. Wind Energy, 20 (3). pp. 411430. ISSN 1099-1824 doi: https://doi.org/10.1002/we.2013 Available at https://centaur.reading.ac.uk/66218/

It is advisable to refer to the publisher's version if you intend to cite from the work. See Guidance on citing.

To link to this article DOI: http://dx.doi.org/10.1002/we.2013

Publisher: Wiley

All outputs in CentAUR are protected by Intellectual Property Rights law, including copyright law. Copyright and IPR is retained by the creators or other copyright holders. Terms and conditions for use of this material are defined in the End User Agreement.

www.reading.ac.uk/centaur 
Central Archive at the University of Reading

Reading's research outputs online 


\title{
Prediction of local wind climatology from Met Office models: Virtual Met Mast techniques
}

\author{
Jessica Standen ${ }^{1}$, Clive Wilson ${ }^{1}$, Simon $_{\text {Vosper }^{1}}$ and Peter Clark ${ }^{2}$ \\ ${ }^{1}$ Met Office, Exeter, UK ${ }^{2}$ University of Reading, Reading, UK
}

\begin{abstract}
The Met Office has developed the Virtual Met Mast ${ }^{\mathrm{TM}}$ (VMM) tool for assessing the feasibility of potential wind farm sites. It provides site-specific climatological wind information for both onshore and offshore locations. The VMM relies on existing data from past forecasts from regional-scale numerical weather prediction (NWP) models, to which corrections are applied to account for local site complexity. The techniques include corrections to account for the enhanced roughness lengths used in NWP models to represent drag due to sub-grid orography, and downscaling methods which predict local wind acceleration over small-scale terrain. The corrected NWP data are extended to cover long periods (decades) using a technique in which the data are related to alternative long-term datasets. For locations in the UK the VMM currently relies on operational mesoscale model forecast data at $4 \mathrm{~km}$ horizontal resolution. Predictions have been verified against observations made at typical wind turbine hub heights at over 80 sites across the UK. In general the predictions compare well with the observations. The techniques provide an efficient method for screening potential wind resource sites. Examples of how the VMM techniques can be used to produce local wind maps are also presented.
\end{abstract}

Keywords: wind resource assessment; wind energy; numerical weather prediction; mesoscale modelling; wind climatology; UK

\section{INTRODUCTION}

Climatological information on wind speed and direction are needed for many applications from major construction projects (e.g. of bridges) and the design of buildings to influencing how trees are planted and where forests should be located [e.g. $1 ; 2 ; 3]$.

The current rapid increase in the use of wind as a clean and renewable energy source is another application of vital importance. For a wind farm to operate successfully and provide a worthwhile power output a minimum average wind speed must be achieved. Additionally if the wind speeds frequently exceed the maximum safe thresholds for the turbines then a site may be unsuitable. Strong turbulence, due perhaps to local terrain or nearby forest, can also affect the viability of wind farm sites.

The traditional Measure-Correlate-Predict (MCP) approach to estimate local wind climatology makes measurements at the site of interest, correlating these with long-term measurements from nearby, and uses this correlation to predict the wind over the period of the long-term data [e.g. $4 ; 5 ; 6]$. A number of different correlation and prediction techniques can be used, with different degrees of sophistication [e.g. 7; 5]. To provide a reliable estimate of wind climatology for a given site, MCP requires:

a) Measurements must be made at the site of interest over a sufficiently long period (e.g. a year) for statistically robust correlations between the measurements and the existing data from nearby. This can be an expensive (and potentially uneconomical) process because ideally the measurements should be at turbine hub height and therefore 
requires either a specialist mast system or a remote sensing approach. The financial cost offshore will be substantially higher.

b) The long-term data at the reference location must be of a consistently high quality, with known instrument faults or changes corrected. The period must be sufficiently long to represent the local climatology. Typically a minimum period of 20 years is required. The reference location should be close to the site of interest, and sufficiently similar in nature that the wind at the two sites is strongly correlated. Clearly, if the winds experienced at the sites are very different (due to local topographic variations, for example) then the method will fail. Modelling techniques can be used to account for the effects of local site complexity but the difficulties of modelling airflow in complex terrain mean this may not be successful $[8 ; 9 ; 10]$

An alternative approach to MCP is to generate synthetic reference climatology data. Over recent years there has been a growing trend of the use of numerical weather prediction (NWP) data for the generation of climatological wind predictions $[4 ; 11]$. Run at sufficiently high horizontal resolution, modern NWP models can, in principle at least, account for local variations in orography and the land surface, and therefore be used to generate site specific wind predictions. One advantage of using model data over reference observations is that model data can be co-located with the target site, avoiding the uncertainties due to using observations at a different site. A recent study [12] shows using model data in place of reference site observations can work well in a MCP approach.

The use of NWP data for climatological wind predictions relies on an accurate model run at high spatial resolution. The resolution requirements will generally depend on the site location. A relatively coarse horizontal grid spacing of a few kilometres may be sufficient for an offshore site, whereas hundreds or even tens of metres may be required over complex terrain. These requirements mean that the modelling must be done over limited areas, using the grid nesting approach typically used for regional NWP whereby the simulations are driven by boundary condition data obtained from a coarser resolution simulation. The need for longterm data for a representative climatology places a severe constraint on what resolutions can be used in practice. Limitations in computer resource may mean that some element of postprocessing of the NWP data will be required in order that local site detail is accounted for.

The purpose of this paper is to describe a method for deriving site-specific wind climatological information from NWP model data and demonstrate how this can provide a useful alternative to the traditional MCP technique. The method forms the basis of the Met Office Virtual Met Mast ${ }^{\mathrm{TM}}$ (VMM) approach, a site-screening tool to provide an initial assessment of a site's potential suitability as a wind farm. The VMM approach produces a site specific time series of wind speed and direction that can be used for a number of applications, such as initial site-screening before conducting a measurement campaign, or as an input for further modelling (including CFD) or investigation. For small wind projects (up to 500kW) the VMM is considered to be a bankable product alleviating the need for at site measurements.

The VMM approach for sites in the UK relies on archived operational Met Office mesoscale forecasts, produced using the Met Office Unified Model (UM) [13]. Between 2006 and 2011, the highest resolution forecasts over the UK used a horizontal grid spacing of $4 \mathrm{~km}$ (the UK4 configuration). Starting in 2011 operational forecasts have also been made using the UKV configuration at a resolution of $1.5 \mathrm{~km}$ [14]. The method described in this paper makes use of both the UK4 and UKV models, along with NWP post-processing techniques developed to correct model winds in complex terrain [15]. These UK4 forecast data have also been assessed for suitability to wind energy applications by [12], who showed that the model winds 
were on average better able to represent the long-term trends at the target sites than nearby observations.

The remainder of this paper is set out as follows. A summary of the UK4 and UKV models and the archived NWP data is provided in Section 2. A set of re-runs of the UK4 model required to extend the archive period, is described in Section 3. The post-processing techniques designed to correct the NWP data and account for local (unresolved) features of the orography are discussed in Section 4. Examples of the VMM method applied at specific locations are presented and compared against measurements in Section 5. Some extensions to the VMM approach are discussed in Section 6 including extending the results using reanalysis data and using the VMM approach to generate regional wind atlases. Conclusions are drawn in Section 7.

\section{MET OFFICE UK OPERATIONAL MESOSCALE MODELS}

The UM is used operationally for both global and limited area NWP as well as climate and coupled atmosphere-ocean Earth-system modelling. The UM is a recognised state-of-the art forecast and climate seamless modelling system [13]. Current operational limited area forecasts which cover the UK are run with horizontal resolutions of 4.4 to $1.5 \mathrm{~km}$. Both $4 \mathrm{~km}$ (UK4) and $1.5 \mathrm{~km}$ (UKV) resolution configurations include data assimilation and use 70 vertical levels, extending up to $40 \mathrm{~km}$. The vertical grid is stretched, with finer resolution in the boundary layer, becoming progressively coarser through the troposphere and stratosphere. The seven model levels below $200 \mathrm{~m}$ above ground level (agl) are 2.5, 13.3, 33.3, 60, 93.3, 133.3 and $180 \mathrm{~m}$.

Limited area configurations are run operationally four times per day, at six hour intervals, except the UKV which runs eight times. Until 2015 a 4km UK only forecast (UK4) was run four times per day with data assimilation and this is currently the main data source for UK locations in VMM, although this is changing to a $4.4 \mathrm{~km}$ resolution hindcast extending back to 1979. In the future, when a suitably long archive is available, this may be replaced by UKV data. Archived UK4 data are available from 2006 onwards. This has been supplemented by running UK4 forecasts for the period 2001-2006, to extend the length of the archive (Section $3)$.

\section{UK4 HINDCAST}

Due to the archive of UK4 data only dating from 2006, forecasts from 2001 to 2006 were rerun to give a $>10$ year set of $4 \mathrm{~km}$ resolution forecasts. As these were not produced in real time, it was not possible to start from Met Office initial conditions, but were initialised from ERA-Interim reanalysis data produced by European Centre for Medium-Range Weather Forecasts (ECMWF) [16]. Reanalysis data initialised a global model every 48 hours, which drove a Western European model and in turn a UK model (Figure 1) with specifications detailed in Table 1. Forecasts from 2001 to 2010 were made, but only the period 2001-2006 is used in the VMM as the archived operational data is used for 2007 onwards. 


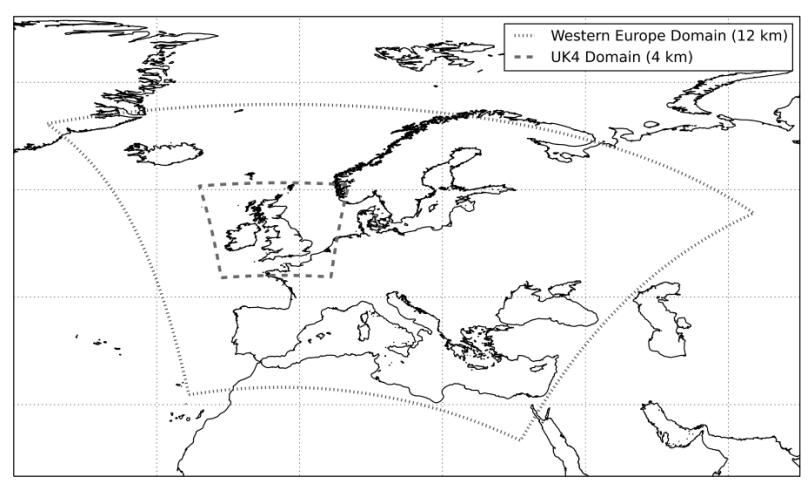

Figure 1: The domains used for the hindcast.

As the UK4 is an operational forecasting model it is continually being developed and upgraded so the archived forecasts are not produced from one consistent model version back to 2007. Conversely the hindcasts were completed using only one model configuration. Due to the lack of data assimilation, different initial data and run lengths, there are differences between the hindcasts and operational forecasts. Four years of overlapping data between the two have been verified against observations available during this period. The reruns have a similar level of accuracy to the operational archived forecasts but, as expected, do not verify quite as well (Table 2). For this reason when operational forecasts are available (2007 onwards) these are used but, despite the slight degradation in accuracy, the hindcast is used between 2001 and 2006 to increase the length of the data used in the VMM. The benefits of using a longer data period outweigh the slight loss in accuracy in the hindcast data.

The following conventions are used for all verification results discussed in this paper unless otherwise stated:

1. When one site has data at different heights, all heights are used and a mean then taken.

2. The "mean of /bias/" is the mean of the absolute values of the station biases.

3. To ensure the results are not dominated by short observation periods, a linear weighting of 0 to 1 is applied as a function of record length up to 360 days, and full weight is given for all observations longer than this. Typical records are between 6 months and 2 years in length. The shortest record was 20 days, with the longest being over 10 years.

\section{POST-PROCESSING TECHNIQUES}

In common with many NWP models, the UM includes physical parameterisations for the effects of unresolved orography. One involves the use of an effective (orographic) roughness length to represent the turbulent form drag exerted on the flow in the boundary layer by subgrid orography. The orographic roughness approach is based on the fact that sufficiently high above the ground, observations of wind profiles over complex terrain resemble those which occur over flat terrain, but with a substantially higher roughness length than that due to the vegetation [17]. The parameterisation involves an estimate of the drag force which would be exerted on the flow by the unresolved hills. This drag is added to the surface stress, which is in turn used to define an effective roughness length. The drag (and hence orographic roughness length) is directly related to the average peak-to-trough amplitude $(H / 2)$ and silhouette area per unit area $(A / S)$ of the sub-grid orography [18]. Within the UM these subgrid parameters are derived from $100 \mathrm{~m}$ resolution terrain datasets.

The parameterisation has been shown to improve NWP performance, through its beneficial impact on the synoptic-scale flow. However, one undesirable consequence of this approach is 
in regions of complex orography where the enhanced roughness causes an excessive reduction in the near-surface wind speed [15]. Unless this is corrected for, the model winds in the lowest $\mathrm{O}(100 \mathrm{~m})$ will have a slow bias and will be unsuitable for wind climatology purposes. This effect is illustrated by Figure 2, which shows mean UK4 forecast wind speeds represented as anomalies from the area average at 33.3, 93.3 and $513.3 \mathrm{~m}$ agl, computed over the period 2008-2011. At 33.3m (Figure 2(a)), regions of significant orography, such as west Scotland, Cumbria (north-west England) and Wales contain greatly reduced wind speeds which are unrealistic. Note that the effect of the orographic roughness decays with height through the boundary layer. At $93.3 \mathrm{~m}$ agl (Figure 2(b)), the very large reductions in wind speed are not as wide spread. Significant decreases in wind speed are present only across the regions of highest terrain (western Scotland). However, it is not until heights of $\sim 500 \mathrm{~m}$ (Figure 2(c)) that the artificial reduction is no longer observed over the most complex terrain.

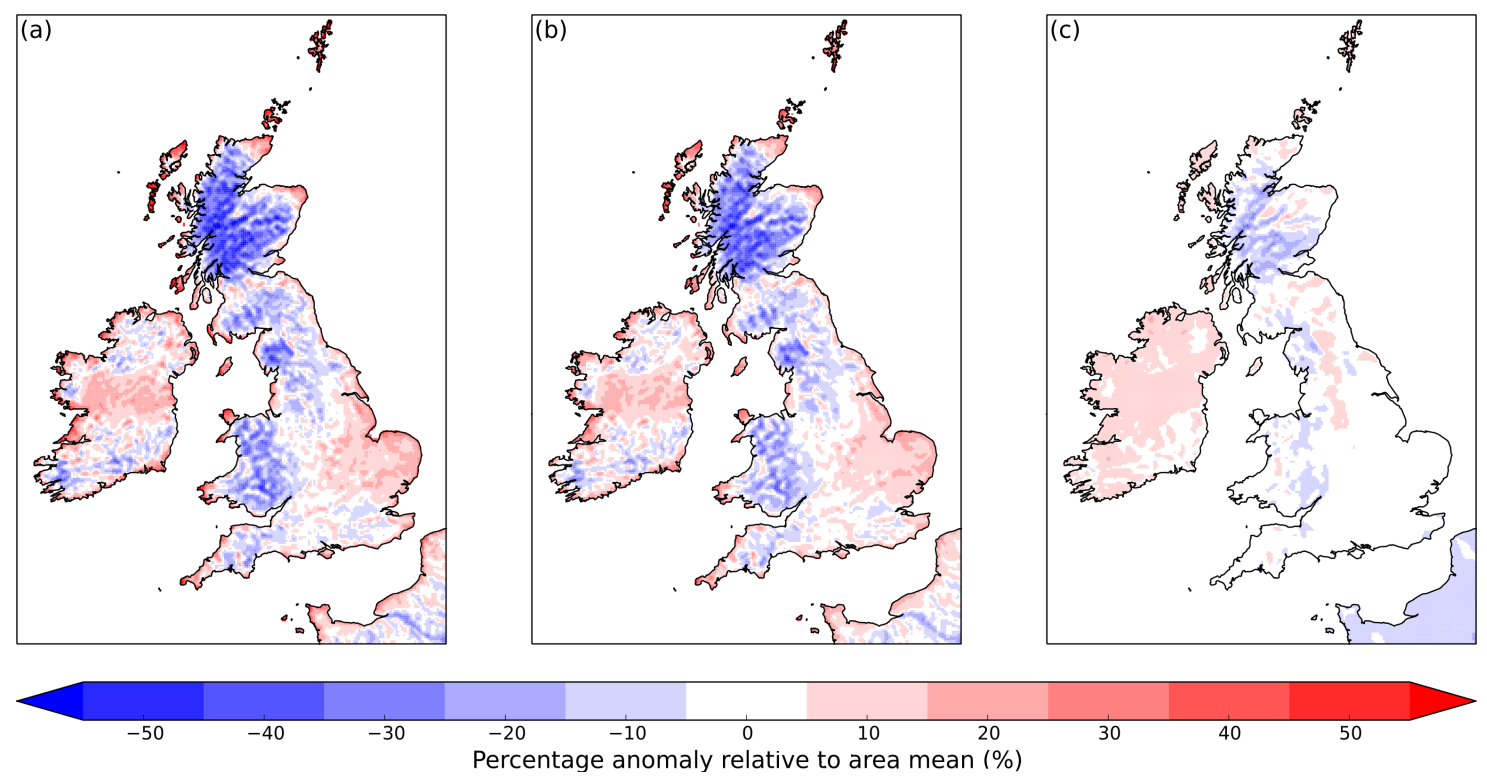

Figure 2: Four-year (2008-2011) mean wind speed anomaly relative to the land area mean (\%) computed from archived UK4 forecasts at a height of (a) 33.3m, (b) $93.3 \mathrm{~m}$ and (c) $513.3 \mathrm{~m}$.

The unphysical reductions in winds must be corrected to estimate wind climatology in areas of complex terrain. The VMM uses a scheme which follows that developed for correcting and downscaling winds in the Met Office nowcasting system [15]. This method consists of two main steps. Firstly a correction is applied to the model winds to account for the enhanced roughness length, increasing the wind speed to a value more consistent with the local vegetative roughness (Section 4.1). Then a downscaling adjustment is applied to account for local acceleration due to orography which is not resolved on the model (4km resolution) grid (Section 4.2). A brief description is given below, with the changes which have been developed for the VMM.

\subsection{Step 1: Orographic roughness correction}

\section{Method 1A: The Howard and Clark (2007) approach.}

Using linear analysis an estimate is made of a reference, or outer-layer height, below which the orographic roughness parameterisation will significantly affect the wind profile. The calculations are based on the $100 \mathrm{~m}$ gridded DTED ${ }^{1}$ data set. For a sinusoidal ridge the ratio

\footnotetext{
${ }^{1}$ The National Imagery and Mapping Agency (NIMA, formerly Defense Mapping Agency (DMA))'s Digital Terrain Elevation Data set
} 
$H /(A / S)$ provides a horizontal length scale, characteristic of a hill wavelength. The height $z_{\text {ref }}$ defined as:

$$
z_{r e f}=a k^{-1}
$$

where $a$ is a tuneable parameter and $k=\pi(A / S) /(H / 2)$ is the horizontal wavenumber, then provides a depth scale over which the hill induced flow perturbations are expected to decay with height in neutrally stratified flow. Equation (1) is the expression used by [15]. It is expected that $z_{\text {ref }}$ will be relatively high over regions of complex orography, because the subgrid peak-to-trough heights $(H / 2)$ will be large and lower over flatter regions. In practice, however, this approach can lead to spuriously high values of $z_{\text {ref }}$ in only modest terrain if $A / S$ is sufficiently small (relative to $H / 2$ ). This is avoided in the VMM implementation by defining limits on some of the parameters as follows:

$$
\begin{gathered}
k=\left\{\begin{array}{l}
\frac{\pi(A / S)}{(H / 2)}, \quad(H / 2) \geq(H / 2)_{\min } \\
\frac{\pi(A / S)}{(H / 2)_{\min }}, \quad(H / 2)<(H / 2)_{\min }
\end{array}\right. \\
\frac{1}{\Delta x_{\max }} \leq \frac{(A / S)}{(H / 2)} \leq \frac{1}{\Delta x_{\min }}
\end{gathered}
$$

and

$$
a= \begin{cases}-\ln \alpha+\ln (k H / 2), & \ln (k H / 2)>\beta \\ -\ln \alpha+\beta, & \ln (k H / 2) \leq \beta\end{cases}
$$

with

$$
0 \leq a \leq 1
$$

where the limiting parameters $(H / 2)_{\min }, \Delta x_{\min }$ and $\Delta x_{\max }$ are set to $1 \mathrm{~m}, 500 \mathrm{~m}$ and $4 \mathrm{~km}$, respectively, $\beta=-4$ and $\alpha$ is a tuning parameter, which defines the proportion to which the hill induced flow perturbations will decay exponentially to (relative to their surface values) between the ground and $z_{\text {ref }}$. Tuning tests in which VMM results have been compared with observations suggest $\alpha=0.12$ works well. The variation of $a, k$, and $z_{\text {ref }}$, as given in Equations (1)-(5), with different values of $A / S$ and $H / 2$ are shown in Figure 3. 

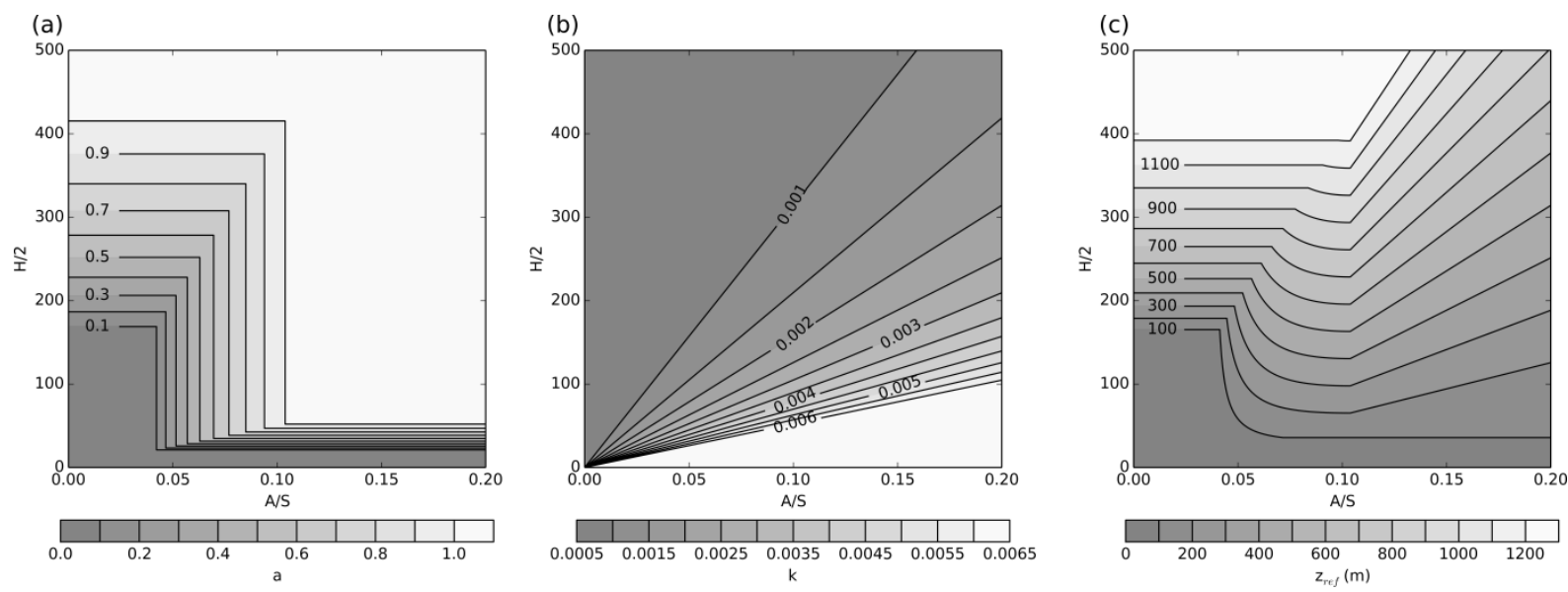

Figure 3: Variation with $A / S$ and $H / 2$ of (a) $a$, (b) $k$ and (c) $z_{r e f}$ as given by Equations (1)-(5).

Figure 4 then shows the values of $z_{\text {ref }}$ calculated over the UK using the method outlined in this section.

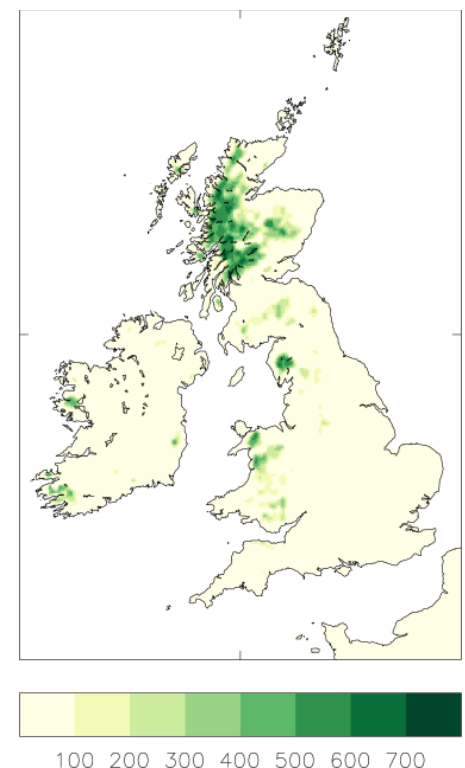

Figure 4: Values of $z_{r e f}(\mathrm{~m})$ calculated from Method 1A over the UK.

Above the reference height the flow is assumed insignificantly affected by the orographic roughness. Where the height for which a wind prediction is required (e.g. the wind turbine hub height) is above $z_{\text {ref }}$, no adjustment is made for orographic roughness. When the prediction height, $z_{\text {hub }}$, is below $z_{\text {ref }}$, a neutral logarithmic wind profile is assumed below $z_{\text {ref }}$ and the roughness corrected wind speed is:

$$
U_{r c}\left(z_{\text {hub }}\right)=U\left(z_{r e f}\right) \frac{\ln \left[\left(z_{\text {hub }}-d\right) / z_{0}\right]}{\ln \left[\left(z_{\text {ref }}-d\right) / z_{0}\right]}
$$

where $z_{0}$ and $d$ are the local vegetative roughness length and displacement height, respectively, which are calculated on a $1 \mathrm{~km}$ grid using a high-resolution land use dataset ( $\sim 100 \mathrm{~m}$ resolution). Details of the calculation of $z_{0}$ and $d$ and hence how they have been used 
at each grid point can be found in [19]. To calculate the area-averaged values used in the VMM the value for the site is calculated from the values of $z_{0}$ and $d$ at the 4 surrounding model grid points. No account is taken here for the effect of non-neutral stability either in calculating $z_{\text {ref }}$ or extrapolating downwards. Further improvements could be made by doing so, but note that $U_{r c}$ is not used directly but only to calculate a scaling factor in order to preserve the diurnal cycle.

\section{Scaling to account for the diurnal cycle}

The correct phase of the diurnal cycle of wind speeds can be lost using the roughness correction. Observations show that reduced stability and greater vertical mixing in the middle of the day results in upper layers exchanging momentum with lower layers. Therefore at lower elevations the diurnal cycle typically has enhanced wind speeds during daylight around midday, whilst at upper elevations the reverse occurs (see Figure 5). In the VMM, using the elevated winds at the reference height $U\left(z_{r e f}\right)$ to correct each hour will reverse the diurnal cycle at lower elevations. This is particularly evident for sites in complex terrain as it is these areas that have the higher values of $z_{\text {ref }}$ so that the roughness correction uses higher level winds. The model reproduces the reversed diurnal phase with height over both flat and complex terrain, although no multilevel high mast data has been found to validate the complex terrain behaviour.

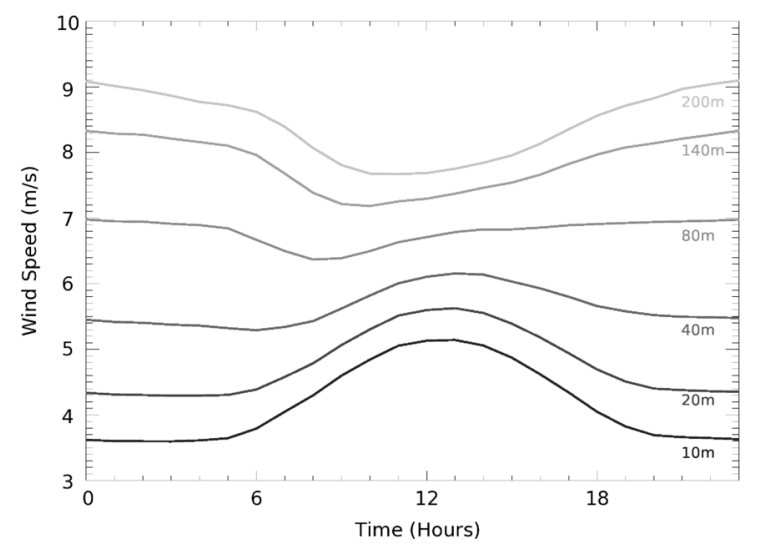

Figure 5: Average diurnal cycle of wind speeds for Cabauw $\left(51.9692^{\circ} \mathrm{N} 4.9258^{\circ} \mathrm{E}\right.$ a low complexity site in the Netherlands) at different elevations over 6 years (2003-2008). Data kindly supplied from the Cabauw Experimental Site for Atmospheric Research (Cesar).

To eliminate this in the VMM a scaling factor is calculated by taking the average ratio of the winds before and after the roughness correction is applied. The average factors are derived for each month. The uncorrected hourly wind time series is then multiplied by these scaling factors; this preserves the mean wind speed from the roughness corrected wind speeds but also preserves the diurnal cycle from the uncorrected winds, which the model is usually able to predict well. This is done for each month in the 10 year time series, although using shorter periods of a few days produces wind speeds of similar accuracy. Henceforth this additional step combined with the roughness correction is referred to as the scaled roughness correction, Method 1As. This empirical procedure has been designed to compensate in part for the neglect of stability in the roughness correction, as the uncorrected winds account for stability through the model's boundary layer scheme.

\section{Method 1B: Use of UKV forecasts to develop a roughness correction}

Since 2011 the Met Office has the operational variable resolution configuration of the UM known as the UKV which has a resolution of $1.5 \mathrm{~km}$ over the UK. The UKV does not contain the orographic roughness parameterisation that reduces the low-level winds over complex 
orography, as verification of the model suggests this would degrade forecast skill and at such high resolution the orography is better resolved. It is possible to use the archived operational UKV forecasts to correct the UK4 forecasts that include this scheme, removing the necessity for Method 1A. Two complete years (2011-2012) of UKV forecasts have been used in a linear regression against the corresponding 2 years of the UK4 forecasts. The linear regression parameters are then applied to the UK4 winds. Different regression factors are derived at each of the model levels from the surface up to a height of 360m. Figure 6 shows the calculated values of the intercept $(\alpha)$ and slope $(\beta)$ at a sample height of $93.3 \mathrm{~m}$ agl.
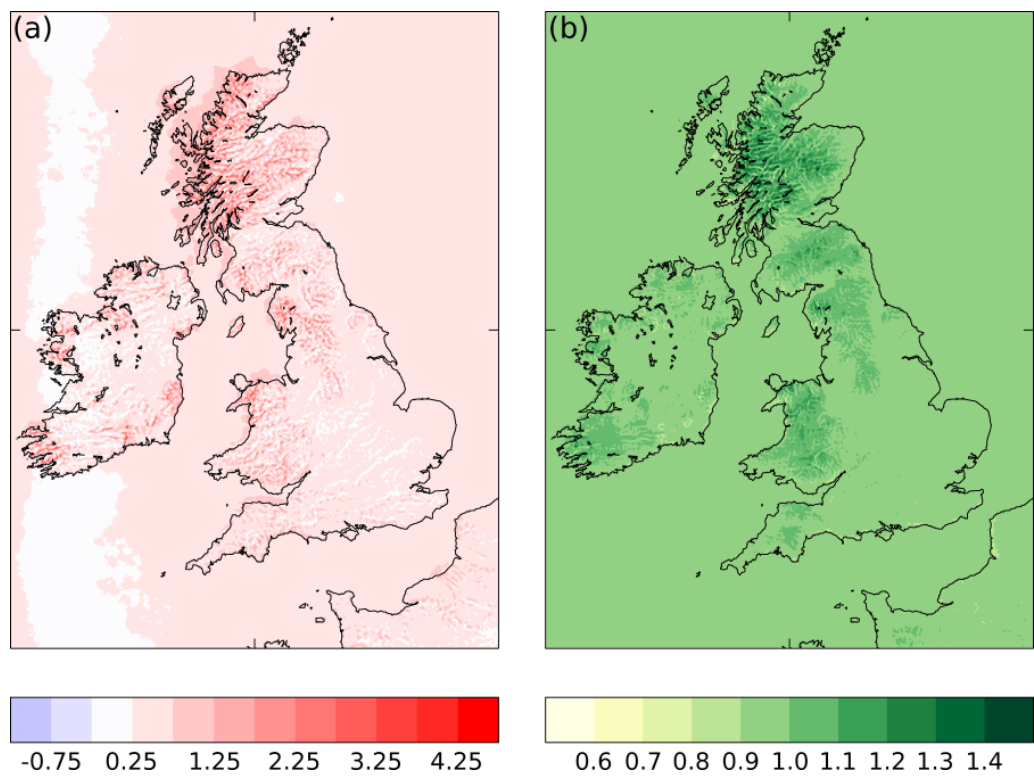

Figure 6: Plots of the regression coefficients (a) $\alpha$ (intercept) and (b) $\beta$ (gradient) at $93.3 \mathrm{~m}$ agl.

The areas with a non-zero value of $z_{r e f}$ in Figure 4, i.e. where the scaled roughness correction is applied correspond well to the areas with the highest $\beta$ values, which is consistent with the highest and most complex orographic sites most affected by the effective roughness parameterisation. Over most of the UK $\beta$ is $\sim 1 \pm 0.1$ and $\alpha$ is small but positive, which shows there is an approximate 1-1 correlation between the wind speeds from the UKV and the UK4. Over most of the UK $\alpha$ being slightly positive is expected as the UKV winds are on average stronger than those from the UK4. Example vertical profiles of $\alpha$ and $\beta$ are shown for a high complexity site, Springhill (further details about this site in Section 5), in Figure 7. As expected the intercept tends to zero and the gradient to 1 at higher heights as the effects of the orographic roughness decreases with height. For lower and medium complexity locations these tend to decay at lower altitudes. Over sea $\alpha$ and $\beta$ are much closer to zero and one everywhere and at all heights. 

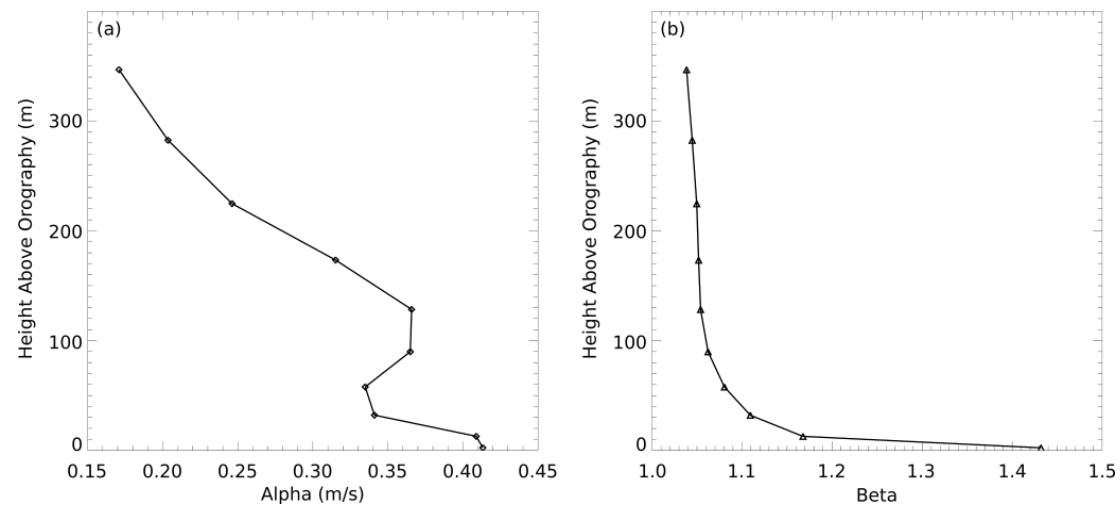

Figure 7: Vertical profiles of the regression coefficients (a) $\alpha$ (intercept) and (b) $\beta$ (gradient) varying with height (above orography) at Springhill.

\subsection{Step 2: Downscaling for sub-grid orography effects}

In addition to the roughness correction described in [15], an adjustment is applied to account for the speed-up effects of unresolved orography.

\section{Method 2A: Simple Howard and Clark (2007) height adjustment}

Following [15], a simple approach involves applying a simplified form of the linear solution for turbulent flow over hills [20] to account for the effect of the difference between the height of the model orography and the true orographic height. In this simple method a reference height is estimated as the inverse horizontal wavenumber of the sub-grid orography and, based on linear theory for the wind perturbations, a velocity perturbation is estimated using Equation 7 of [15]. This perturbation is applied to the scaled roughness corrected wind speed. Initially this method was used in the VMM, but this has since been replaced by a more sophisticated technique (see Method 2B, below).

\section{Method 2B: A 3D linear model adjustment}

An alternative and more sophisticated approach is to derive the full three-dimensional linear solution for turbulent flow over the local terrain and use this to adjust the roughness scaled wind predictions. The method applied in VMM, which replaces Method 2A, is based on 'model D' of [20]. This is a steady-state quasi-analytic solution for neutrally stratified turbulent boundary layer flow. The solution is obtained by taking a two-dimensional discrete Fourier transform of the terrain height data and solving for each Fourier component of the horizontal velocity perturbation. Unlike Method 2A, this approach avoids the need to estimate a single length scale for the orography, and also allows directional effects to be accounted for.

For a given site the terrain data is extracted from the 100m gridded DTED data set over $25.6 \times 25.6 \mathrm{~km}$ area centred on the site. To avoid spurious effects from periodicity (implicit in the use of a discrete Fourier transform) a tapering function is applied to the terrain data:

$$
w(r)=0.5 \times\left(1+\cos \left(\frac{2 \pi r}{R}\right)\right)
$$

where $r$ is the distance from the site and $R$ is half the domain width (i.e. $12.8 \mathrm{~km}$ ).

Model D solutions are then computed for 72 equally spaced wind directions, and a separate speed-up factor, $\Delta s$ is computed for each direction at each required elevation,

$$
\Delta s=\frac{\left|\mathbf{U}_{\mathbf{D}}\right|-\left|\mathbf{U}_{\mathbf{0}}\right|}{\left|\mathbf{U}_{\mathbf{0}}\right|}
$$


where $\mathbf{U}_{\mathbf{D}}$ and $\mathbf{U}_{\mathbf{0}}$ are the model $\mathrm{D}$ and unperturbed horizontal velocity, respectively. A direction-height look-up table for $\Delta s$ is then used to adjust the scaled roughness wind speed time series to account for the local orography.

\section{COMPARISONS WITH OBSERVATIONS}

The VMM has been extensively verified based on a large sample of 211 unique location-elevation pairings across 92 sites in the UK giving 175 site-years of observations. The observations comprise of a combination of meteorological mast data on prospective sites and Nacelle data from turbines in operation.

Each site is classified as offshore, near-shore, low, medium, or high complexity based on the values of $A / S$ (silhouette area per unit area) computed from the $100 \mathrm{~m}$ terrain data. The categories are defined as follows:

$$
\text { complexity }= \begin{cases}\text { offshore, } & A / S=0 \& D>5 \mathrm{~km} \\ \text { near-shore, } & A / S=0 \& D \leq 5 \mathrm{~km} \\ \text { low, } & 0<A / S<0.0075 \\ \text { medium, } & 0.0075 \leq A / S<0.02 \\ \text { high, } & 0.02 \leq A / S\end{cases}
$$

where $D$ is the distance to the nearest land.

Figure 8 shows time series of monthly mean wind speeds at 3 sites: Springhill, a high complexity hill-top location in Shropshire, UK, where a 30m turbulence mast was deployed for $\sim 1$ year [21]; Cardington, a low complexity site in Bedfordshire with over 7.5 years of data; and Cabauw, a low complexity site in the Netherlands with 11 years of observations. The VMM results replicate the observations reasonably well with a high degree of correlation ( $\mathrm{R}^{2}$ values are 99, 95 and $96 \%$ respectively.). 

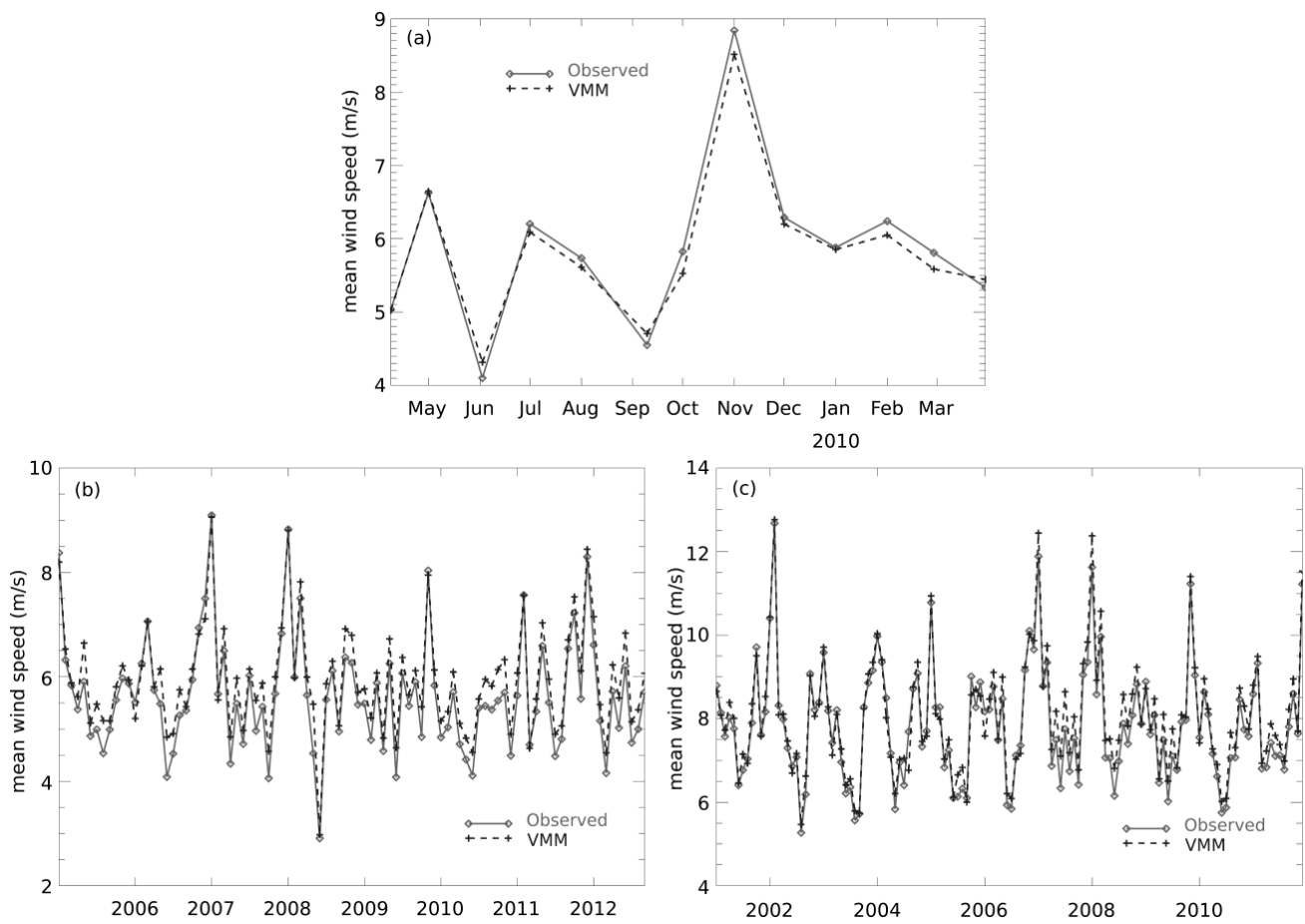

Figure 8: Monthly mean wind speeds at (a) Springhill 30m, (b) Cardington 50m and (c) Cabauw 140m. VMM has had Methods 1As and 2B applied.

Figure 9 shows the monthly mean bias and RMSE at the different stages of the VMM process for Springhill. At this site there is a modest improvement in both the bias and RMSE when the scaled roughness correction is applied, the height adjustment then increases the wind speeds, but too much, so there is now a positive bias, but an improvement in the RMSE.

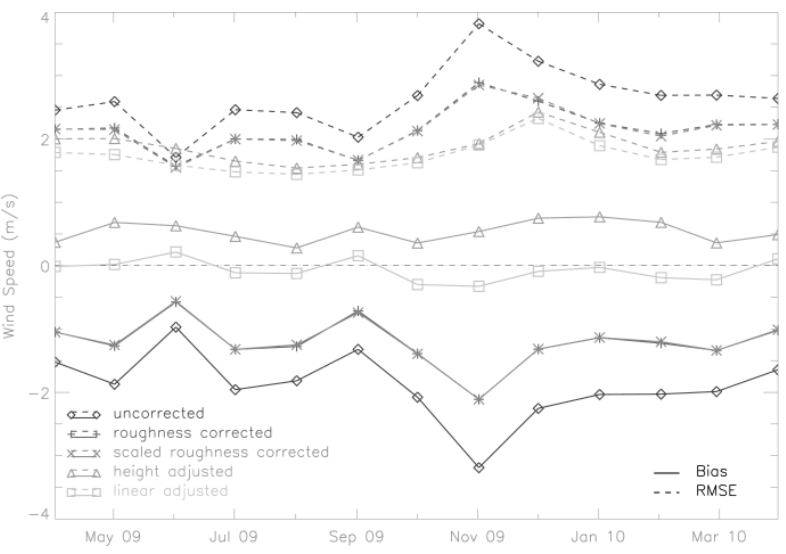

Figure 9: Wind speed bias and RMSE at Springhill at 30m, shown for the different correction methods and stages. It should be noted that the roughness corrected line is plotted, but by the nature of the scaled roughness correction, the monthly mean wind speeds are preserved from the roughness corrected winds, so the scaled roughness corrected line lies on top of the roughness corrected line.

At this site the surrounding orography (Figure 10) is very complex and the simple height adjustment fails to allow for this properly. The 3D linear model takes better account of the orographic complexity, increasing the scaled roughness wind speeds, but to a lesser extent, causing the bias to be almost zero, and reducing the RMSE further. 


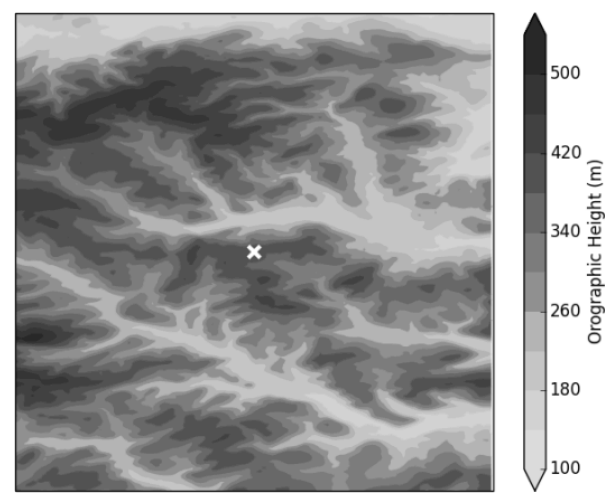

Figure 10: High resolution orography showing the terrain around Springhill (cross).

Table 3 shows the correlation $\left(\mathrm{R}^{2}\right)$ between the observed and modelled wind speed at the different stages of the VMM process. The $\mathrm{R}^{2}$ values for uncorrected, scaled roughness corrected (1As) and UKV corrected (1B) winds are 65.7-66.6\%; applying the height adjustment to the scaled roughness corrected winds ( $1 \mathrm{As} \& 2 \mathrm{~A}$ ) improves $\mathrm{R}^{2}$ by $0.5 \%$; however applying the 3D linear model (1As\&2B) instead improves $\mathrm{R}^{2}$ further to $68.4 \%$ and using the UKV and linear adjustment (1B\&2B) the $\mathrm{R}^{2}$ is slightly lower at $67.8 \%$. When looking at the monthly $\mathrm{R}^{2}$ the correlations are very high at all stages. The $\mathrm{R}^{2}$ for the mean diurnal shows the requirement for the scaling following the roughness correction (1A), and that if the height correction (2A) was used a scaling would also need to be applied there.

Figure 11 shows hourly wind speeds for VMM, when both the scaled roughness correction (1As) and 3D linear model adjustment (2B) have been applied, plotted against observations at Springhill. Mostly there is a good agreement between VMM and the observations. For 28\% of cases the VMM wind speed is within $10 \%$ of the observed and similarly $51 \%$ are within $20 \%$ of the observed. For $47 \%$ of cases the VMM wind speed is within $\pm 1 \mathrm{~ms}^{-1}$ of the observed, and $78 \%$ are within $\pm 2 \mathrm{~ms}^{-1}$ of the observed.

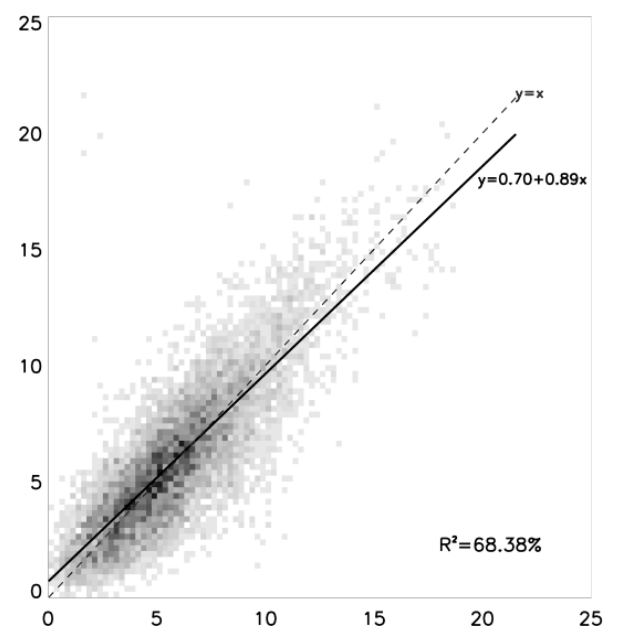

Figure 11: Fully corrected VMM hourly wind speeds $\left(\mathrm{ms}^{-1}\right)$ against observations at Springhill 30m, where the shading of the square represents the frequency of points in that area (darker colour means more points).

As discussed in Section 4.1 (Figure 5) the average diurnal cycle of the wind speeds can be of opposite phase to that at lower levels, which can lead to a reversal in the diurnal cycle at lower levels when the upper level winds are brought down in the 1A roughness correction (maximum winds at night, minimum winds in the middle of the day). The scaled roughness correction (1As) corrects this, and an example of the diurnal cycles at $30 \mathrm{~m}$ at Springhill is 
shown in Figure 12. The shape and amplitude of the observed diurnal cycle has been replicated by the uncorrected model winds. However there is an offset in the values. The scaled roughness correction (1As) preserves the diurnal variation and the 3D linear model scaling (2B) brings the VMM winds into close agreement with the observed values. In contrast the direct application of the roughness correction (1A) distorts the diurnal variation which is further distorted by the height correction $(2 \mathrm{~A})$.

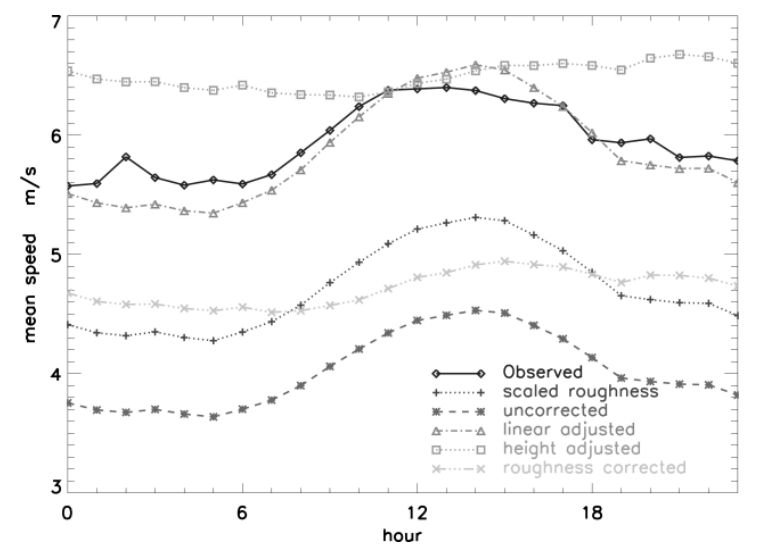

Figure 12: Average diurnal cycle at Springhill $(30 \mathrm{~m})$ for observations and VMM corrected winds.

A wind rose comparison of 30m winds at Springhill (Figure 13) shows the VMM successfully captures the main frequency and strength distribution in the twelve directional bins, with a slight veering away from the observed in the south-westerly bins.

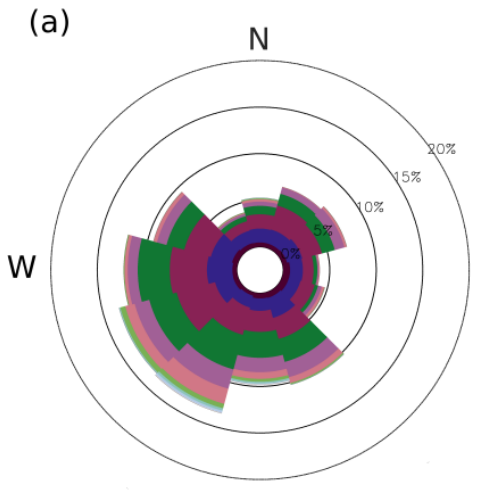

S

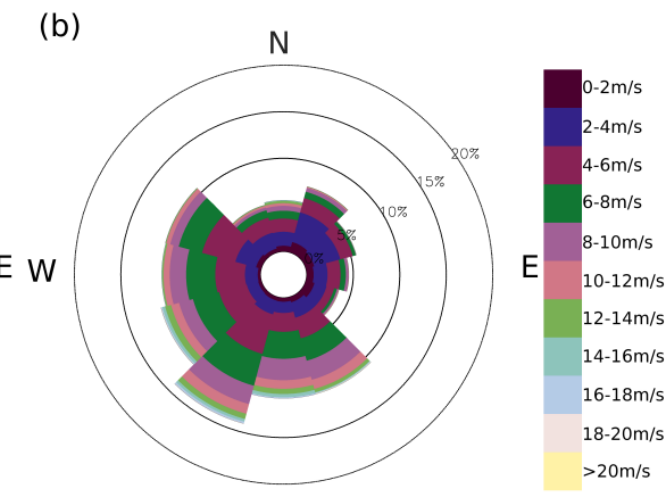

S

Figure 13: Wind roses at Springhill for (a) 30m downscaled model distribution and (b) observed.

An accurate distribution of wind speed is needed to estimate the expected wind power at a site as the power depends on the wind speed cubed. At Springhill the fully corrected and adjusted wind distribution (Figure 14(b)) agrees well with the observed distribution (Figure 14(c)), satisfying a Kolmogorov-Smirnov test of fit at 5\% significance. In contrast, the uncorrected winds (Figure 14(a)) are a much poorer representation of the observed due to errors in both location and spread. 

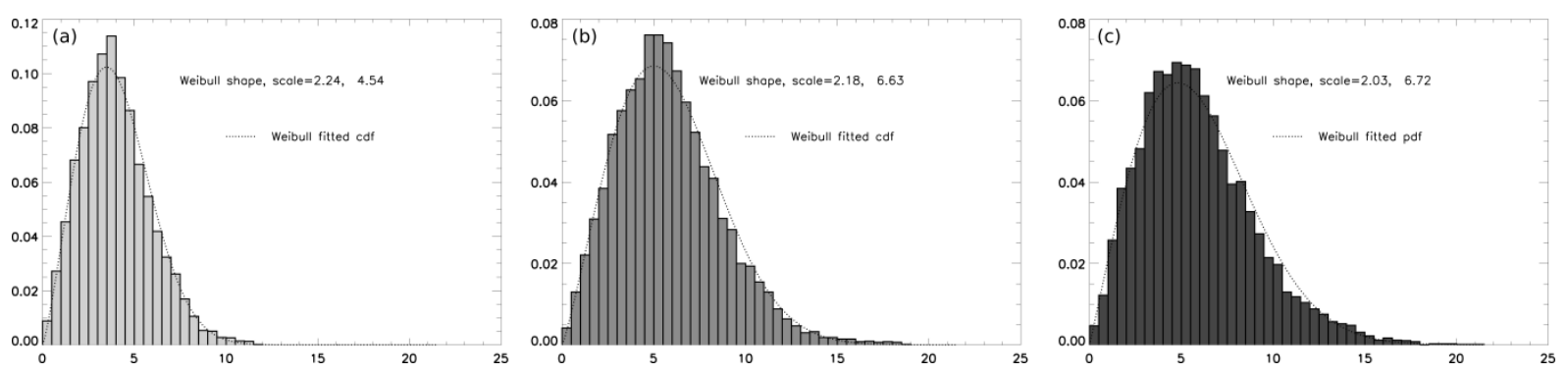

Figure 14: Wind speed distributions for Springhill at $30 \mathrm{~m}$ for (a) uncorrected, (b) fully corrected and (c) observed.

Due to the commercial sensitivity of most of the data used to verify the VMM, it is possible to show the site specific results only for a limited number. Table 5 shows the breakdown of how all the sites are distributed across the complexity classes both on land (76) and offshore (16). These are spread geographically across the UK, as shown later in Figure 16, however only 86 of them are within the UKV domain.

Table 4 is a summary of verification statistics across each stage of the adjustment process across all sites where data are available. In addition to the different stages and corrections of the VMM the statistics of using just the ERA-Interim reanalysis data, interpolated to each site location and height, are also shown to demonstrate the large improvement due to the downscaling (uncorrected compared to reanalysis), as well as the smaller improvements from each of the different correction steps. Mean $\mathrm{R}^{2}$ values for hourly, monthly and diurnal means are 72,91 and $87 \%$ compared to 71,88 and $72 \%$ for the reanalysis, with small changes from the post-processing except for the roughness and height corrected, as for Springhill (Table 3).

The VMM performance for the fully corrected results (1As\&2B) for different complexity (Table 5) shows the offshore and near-shore sites have smallest errors and the medium and high complexity sites have the largest errors as expected. The relatively small mean biases indicate there is no overall systematic bias from the NWP plus adjustments.

Observations at a variety of heights from 10 to $200 \mathrm{~m}$ above ground/sea level are used. Figure 15 shows the mean bias and standard deviation around the mean for all of the location elevation pairings. Observations are split into $10 \mathrm{~m}$ bins (Figure 15(a)) and are also averaged (Figure 15(b)). Note Figure 15(a) only shows results for heights between 10 and 100m, whereas Figure 15(b) shows the result for heights between 10 and $200 \mathrm{~m}$. The reason for not including the results between 100 and $200 \mathrm{~m}$ in Figure 15(a) is due to the small number (4) of measurements available above $100 \mathrm{~m}$.
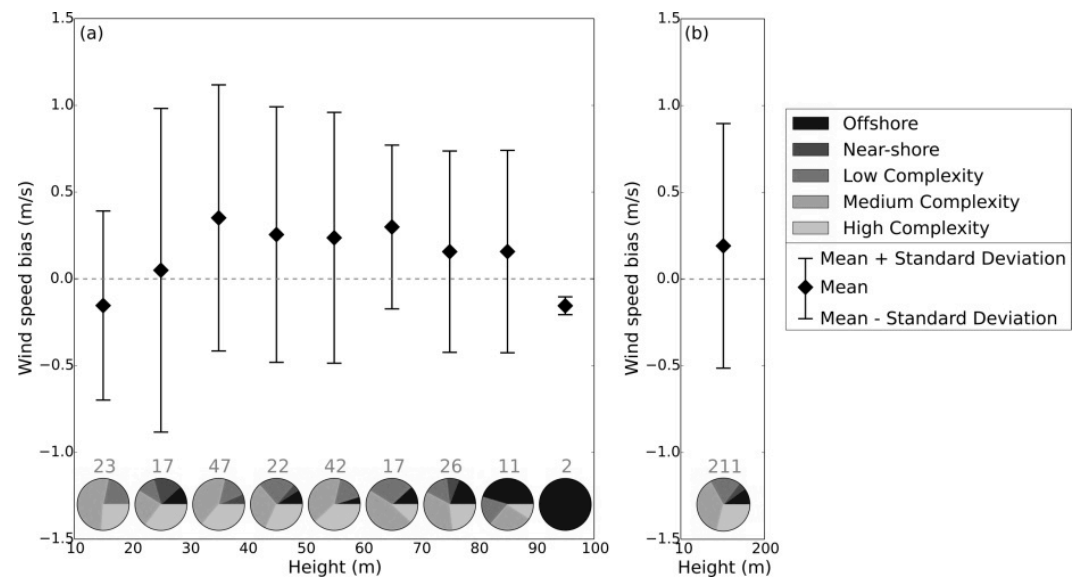

Figure 15: Bias (a) in 10m height bins (10-100m) and (b) over all heights (10-200m) - frequency of each bin (in grey) and a pie-chart of the complexity classifications for each bin. 
It might be expected that with increasing hub height the VMM would perform better as the local surface has a lesser effect on the winds. Figure 15 possibly reflects this but the results are not statistically significant. Each height bin has a different frequency distribution of site classification hence it is not possible to separate out the effects of hub height from site complexity. Many more observations would be required to do this.

The average bias in the VMM compared to the observations is plotted spatially in Figure 16. This shows that there is a small spatial bias variation in the VMM with a tendency to overestimate in southern UK and under-estimate in northern UK. The largest over-estimation is for Wales, but as there are relatively few observation sites the results should not be regarded as significant; the impact of sampling will be high.

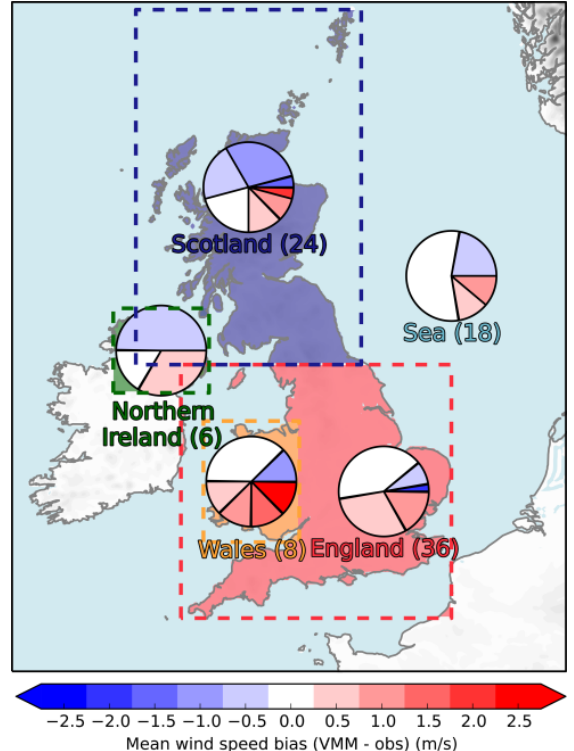

Figure 16: The mean biases between VMM predicted and observed wind speeds at a basket of 92 sites, with hub heights between 10 and $200 \mathrm{~m}$ agl. The distribution of these biases has been plotted as pie charts for land sites in 4 areas approximately based on countries and one for sea. Where there is more than one height available for a station an average over the heights has been taken and that value of the bias used. The number in brackets refers to the number of sites in that region.

Table 6 shows the same statistics as Table 5 split by complexity type using the alternative orographic correction by UKV scaling combined with the linear adjustment (1B\&2B). Comparison of the two tables shows over all sites similar accuracy is obtained for the standard deviation. Results for complexity types differ more, with standard deviation better using UKV for all except low complexity; mean biases are usually worse using UKV.

\section{EXTENSIONS TO THE VMM METHOD}

This paper has outlined and verified the basic VMM method. Additional extensions are described in this section. The VMM results are extended to establish a longer period climatology (Section 6.1) and wind maps showing the relative local wind speeds about the site are also produced (Section 6.2). Finally the VMM approach can be extended on a grid to generate wind atlases (Section 6.3).

\subsection{Extension of time series to establish a longer period climatology}

Although the UK4 archive data set has been extended through hindcasts (Section 3) the base $4 \mathrm{~km}$ data still only covers from 2001 to the present day. It is desirable to extend the climatology to a longer period by establishing a relationship between ERA-Interim reanalysis data and the VMM time series. ERA-Interim is available from 01 January 1979 to 
approximately present day at a T255 spectral horizontal resolution which corresponds to approximately $79 \mathrm{~km}$. A conditional probability matrix is used to relate the VMM hub-height winds to those of ERA-Interim reanalysis over the common period of 2001 to the present day. The matrix is then applied to the distribution of winds in ERA-Interim over the longer ERA period to derive an implied longer term climatology of hub height winds. This is an extension of the method sometimes used in MCP [22].

Time series of winds, $\mathbf{u}_{c}$, are extracted from ERA-Interim at a $60 \mathrm{~m}$ nominal height for the full period. These winds are related to the downscaled winds from the VMM at the hub height, $\mathbf{u}_{h u b}$, using a matrix method. A probability matrix is calculated with a wind speed bin size of $1 \mathrm{~ms}^{-1}$ and $30^{\circ}$ wind direction bins for the overlapping period between $\mathbf{u}_{h u b}$ and $\mathbf{u}_{c}$. The element $(l, k)$ of the matrix is the probability of $\mathbf{u}_{h u b}$ in bin $k$ when $\mathbf{u}_{c}$ lies in bin $l$ which is given by:

$$
\mathbf{P}_{k l}=\mathbf{P}\left(\mathbf{u}_{h u b, k} \mid \mathbf{u}_{c, l}\right)
$$

The wind probability distribution over the full period at hub height is given by the matrix multiplication

$$
\mathbf{P}_{k}=\sum_{l=1}^{480} \mathbf{P}_{k l} \mathbf{P}_{l}
$$

where $\mathbf{P}_{l}$ is the wind distribution of $\mathbf{u}_{c}$ over the full period. If a bin is not populated by the 10 years of overlapping data a one-to-one correspondence is assumed. The 10 years of overlap samples all wind direction bins, but not all the higher wind speed bins (that are present, albeit rarely, in the long period) may be sampled. The one-to-one correspondence could result in slight over/under-estimation of the long-term mean wind speeds, depending on the relative occurrence of the unsampled wind speeds and the difference between the hub level wind speeds and the $60 \mathrm{~m}$ nominal ERA wind speeds. An alternative assumption of zero probability for the unpopulated bins results in typical differences of $0.01 \mathrm{~ms}^{-1}(0.02 \%)$ between the long term means as typically only $0.1 \%$ of the joint probability bins are unpopulated.

The probability matrix and wind distributions permits simultaneous and consistent adjustment for differences in both wind direction and wind speeds between the VMM downscaled winds and the ERA-Interim winds. This has two major advantages compared to traditional linear regression methods; in the latter, directional changes are harder to treat properly, and so may for example compromise the accuracy of the long term wind rose. This means locally forced flow that may be better represented at $4 \mathrm{~km}$ resolution will be more accurately reflected in the long term climatology even though it may be poorly captured or not at all by the coarser resolution ERA-Interim reanalyses. Also the adjustment for wind speed bias between the ERA-Interim and VMM winds is not constrained to be strictly linear, so that different wind speed ranges may be adjusted differently.

An example of this procedure is shown in Figure 17 for Springhill. This shows how the directional and speed differences of the ERA-Interim 60m data (Figure 17(b)) are transformed to be more similar to the original downscaled VMM distribution (Figure 17 (a)) in the final derived distribution (Figure 17(c)). The mean wind speed is increased in the final derived distribution compared to the original downscaled model distribution to reflect that the years used in the VMM were less windy than over the whole climatological period. For larger differences between the short term VMM distribution and the corresponding period of ERAInterim more radical changes would be expected. 

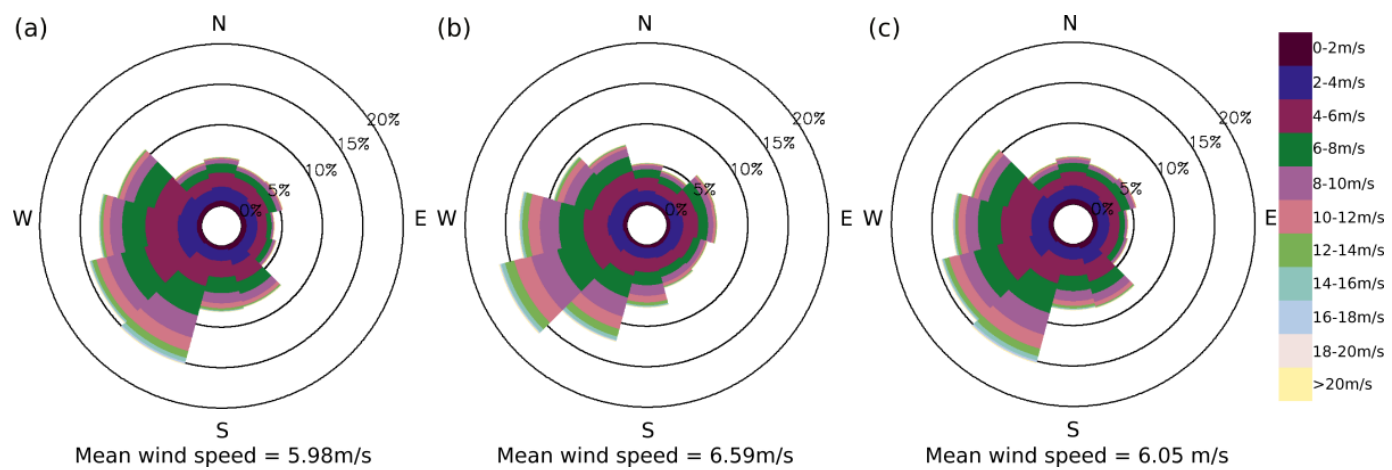

Figure 17: Wind roses at Springhill for (a) 30m downscaled model distribution, (b) 60m ERA-Interim long period and (c) $30 \mathrm{~m}$ derived model distribution.

\subsection{Wind Speed Maps}

A mean wind speed map for a few $\mathrm{km}$ around a site can be derived from the VMM by using the probability distribution for hub wind speed and direction, $P\left(U_{s}, \theta_{j}\right)$ from one site. For a given wind direction, $\theta_{j}$, the mean wind speed map $\bar{U}\left(x, y, \theta_{j}\right)$ can be derived from the probability density function for the wind speed at the site, $U_{s}$, by

$$
\bar{U}\left(x, y, \theta_{j}\right)=\sum_{i} U_{s_{i}} F\left(x, y, \theta_{j}\right) P\left(U_{s_{i}}, \theta_{j}\right)
$$

where

$$
F\left(x, y, \theta_{j}\right)=\frac{1+\Delta s\left(x, y, \theta_{j}\right)}{1+\Delta s\left(\text { site }, \theta_{j}\right)}
$$

and $\Delta s\left(x, y, \theta_{j}\right)$ is given by the spatial extension of Equation (8).

Hence, the overall mean wind speed map averaged over all wind direction is defined as:

$$
\bar{U}(x, y)=\sum_{j} \sum_{i} U_{s_{i}} F\left(x, y, \theta_{j}\right) P\left(U_{s_{i}}, \theta_{j}\right)
$$

An example wind map is shown for the region around Springhill at $30 \mathrm{~m}$ in Figure 18. Similar wind maps are also produced for the climatological extension (Section 6.1 using $\mathbf{P}_{\mathbf{k}}$ Equation (10)). 


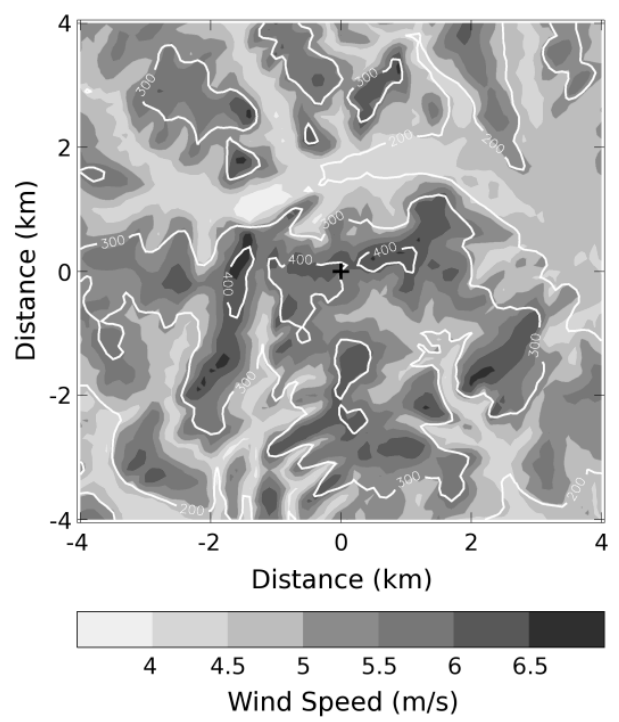

Figure 18: Example wind speed map for Springhill at 30m. Contours denote terrain height; cross denotes the location of the Springhill mast.

These maps only account for the relative speed differences caused by orographic variation around the site, and do not take into consideration changes in surface roughness for example. Despite this limitation they help to set the VMM site wind estimate in context and show the variation that may be expected in the vicinity.

\subsection{Wind atlases}

The VMM technique can be adapted and applied on a grid to generate wind atlases to establish the wind climatology over a wide area, for example the updated Irish national onshore and offshore wind atlas [23]. It is possible to utilise the well developed and verified site specific method to generate a wind atlas which improves on the often used method of simply averaging raw model winds.

Although it would be possible to use the full VMM method some simplifications and adaptations were made when generating the updated Irish wind atlas, for example using fewer wind directions in the linear model (2B), made for computational efficiency, and using a different land use dataset. Full details of the method can be found in [23].

An example 50m agl wind atlas over parts of Northern Ireland at the different stages is shown in Figure 19 on a $1 \mathrm{~km}$ grid, generated using the hindcast data spanning 2001-2010. Figure 19(a) shows the raw $4 \mathrm{~km}$ (i.e. uncorrected) forecast data interpolated to $50 \mathrm{~m}$ agl and onto a $1 \mathrm{~km}$ grid for comparison purposes. Figure 19(b) shows the equivalent atlas once the scaled roughness correction (1As) has been applied and the final atlas is shown in Figure 19(c) where the 3D linear model correction (1As\&2B) has been applied. The difference between the uncorrected and the fully corrected is presented in Figure 19(d), which shows how over most areas the corrections increase the mean wind speed at $50 \mathrm{~m}$ over Northern Ireland, particularly over areas of complex orography (e.g. Sperrin (north-west) and Mourne (southeast) Mountains). 

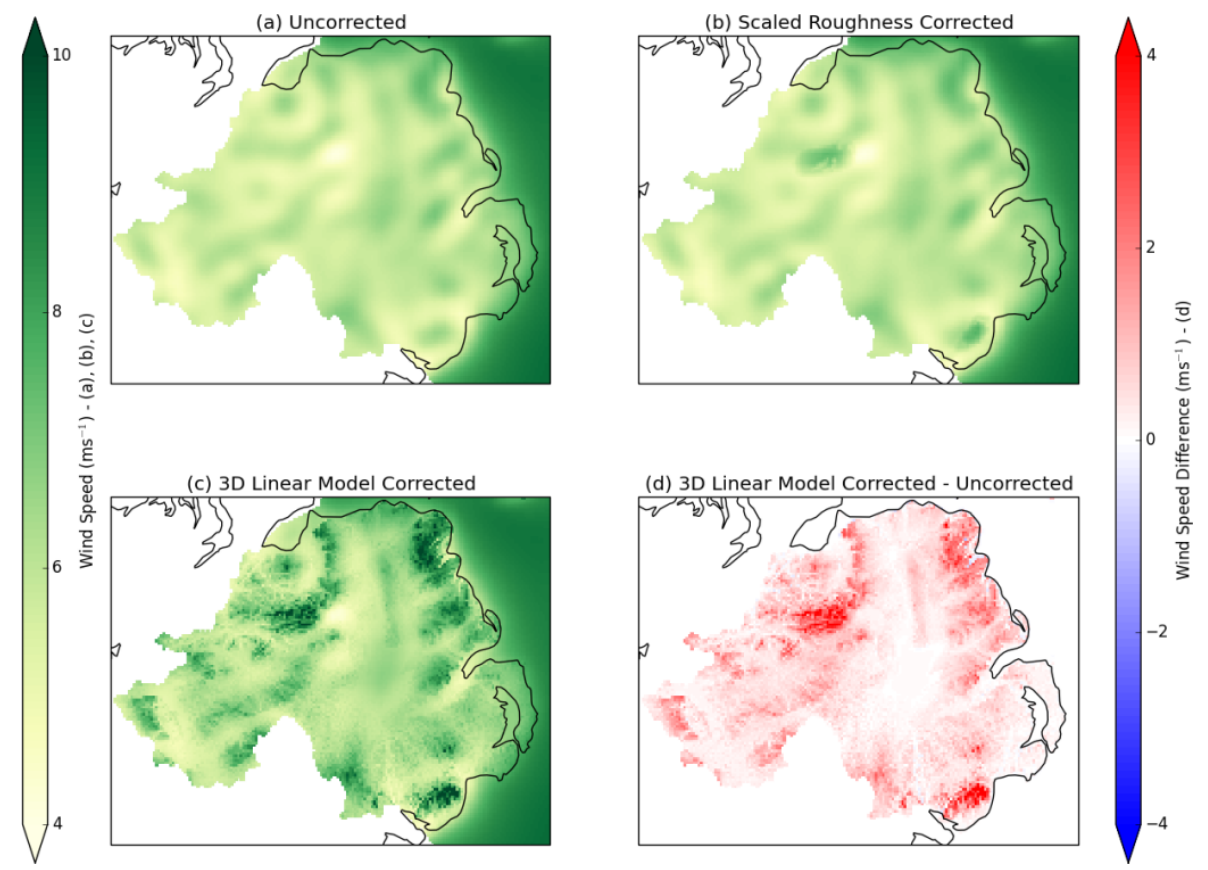

Figure 19: Mean wind speed over Northern Ireland at 50m agl for the different steps (a-c) and the difference between the fully corrected mean wind speed and the uncorrected (d).

\section{CONCLUSIONS}

Archived model forecasts are a valuable resource for predicting wind climatology, particularly when local observations are not available at a comparable height. The VMM makes use of these data and applies corrections to account both for the excessive deceleration of model winds over complex orography due to the orographic drag parameterisation, and the height differences between the model and real terrain. It provides a cost-effective alternative to on-site monitoring.

We have shown that using the roughness correction based on [15] with the addition of a scaling (1As) to preserve the diurnal cycle can correct for the effect of the orographic drag, and reduce the artificial reduction in the near-surface wind speed. Then applying a 3D linear model adjustment (2B; based on [20]) to these winds can account for the local height differences between the smoothed model and high resolution terrain at the site. This paper has shown that the resulting corrected time series of winds agree well with the observed wind speeds at a large number of sites with varying complexities, locations and heights. The range in mean biases across the complexity types is $-0.02 \mathrm{~ms}^{-1}$ (offshore) to $0.18 \mathrm{~ms}^{-1}$ (medium complexity) and the standard deviations of the biases ranging from $0.23 \mathrm{~ms}^{-1}$ (offshore) to $0.98 \mathrm{~ms}^{-1}$ (high complexity).

Using UKV $1.5 \mathrm{~km}$ model to correct for the orographic roughness (1B) in place of 1 As yields a similar level of accuracy, with mean bias ranging from $-0.41 \mathrm{~ms}^{-1}$ (near-shore) to $0.36 \mathrm{~ms}^{-1}$ (medium complexity) and the standard deviations of the biases ranging from $0.10 \mathrm{~ms}^{-1}$ (offshore) to $0.93 \mathrm{~ms}^{-1}$ (high complexity).

To establish a longer period climatology, the VMM time series is extended using a probability matrix method and ERA-Interim data. This enables the wind speed distributions and wind roses to be adjusted for the differences in the climatology between the years used in the VMM and those in ERA-Interim. 
Traditionally wind maps around a site have relied on interpolated observations at $10 \mathrm{~m}$ and statistical techniques; however this has been replaced on the whole by using microscale models of varying complexities including linear models and CFD. These microscale models are often expensive, and by using the methods as outlined in this paper it is possible to generate wind maps by post-processing model data, offering an alternative approach which has a physical basis. The methods outlined in this paper do not replace on-site measurements nor rigorous site assessments such as microscale modelling, however it provides a cost effective initial site screening tool and for small wind projects (up to $500 \mathrm{~kW}$ ), where microscale modelling and measurement campaigns can be prohibitively expensive, can provide a bankable alternative report. In addition to applying the VMM for individual sites it is possible to apply the same methods to gridded forecast data to produce wind atlases.

While the post-processing corrections in VMM clearly improve the accuracy of the wind predictions relative to raw model data, it is likely that further improvements could be developed. For example, although the underlying mesoscale model (UM) accounts for subgrid variations in land surface properties (through the use of a tiled land surface scheme, [24]) these are averaged by the surface exchange scheme to provide grid-box mean fluxes and the VMM methodology does not correct for these variations locally. The representation of local variations in surface roughness, due to urban or forested areas for example and accounting for directional fetch, may further improve the VMM predictions; however the importance of such effects will depend on the hub height for which estimates are required, relative to the scale height over which local roughness variations affect the local wind profile (the blending height). For variations in roughness with a wavelength of $4 \mathrm{~km}$ (the UK4 model grid length), the blending height is likely to be around 20m [25] suggesting that this effect is more important for wind predictions at relatively low hub heights. Further improvements might also come from the inclusion of stability effects in both the orographic roughness correction step and the 3D linear model.

For wind energy applications the VMM can be used as an efficient method for the initial assessment of a large number of potential sites. Although the VMM was initially developed for wind energy applications there are many other applications this technique could be used for, from forestry to construction.

\section{ACKNOWLEDGEMENTS}

The authors thank Graeme Candlish, Leo Hume-Wright, Alasdair Skea, Karen Walter and Stuart Webster (Met Office) for their input into the developments and verification of the VMM over the years. The data for Cabauw was kindly supplied from the Cabauw Experimental Site for Atmospheric Research (Cesar). We also wish to thank the three anonymous reviewers for their very helpful comments which have challenged us to clarify the description and look deeper at the behaviour of the VMM.

\section{REFERENCES}

1. Gardiner, BA, Quine, CP. Management of forests to reduce the risk of abiotic damage - a review with particular reference to the effects of strong winds. Forest Ecology and Management 2000; 135(1-3):261-277, doi:10.1016/S0378-1127(00)00285-1.

2. Diana, G, Yamasaki, Y, Larsen, A, Rocchi, D, Giappino, S, Argentini, T, Pagani, A, Villani, M, Somaschini, C , Portentoso, M. Construction stages of the long span suspension Izmit Bay Bridge: Wind tunnel test assessment. Journal of Wind Engineering and Industrial Aerodynamics 2013; 123, Part B:300-310, doi:10.1016/j.jweia.2013.09.006. 
3. Huang, MF, Li, Q, Chan, CM, Lou, WJ, Kwok, KCS , Li, G. Performance-based design optimization of tall concrete framed structures subject to wind excitations. Journal of Wind Engineering and Industrial Aerodynamics 2015; 139:70-81, doi:10.1016/j.jweia.2015.01.005.

4. Landberg, L, Myllerup, L, Rathmann, O, Petersen, EL, Jørgensen, BH, Badger, J , Mortensen, NG. Wind Resource Estimation-An Overview. Wind Energy 2003; 6(3):261-271, doi:10.1002/we.94.

5. Carta, JA, Velázquez, S, Cabrera, P. A review of measure-correlate-predict (MCP) methods used to estimate long-term wind characteristics at a target site. Renewable and Sustainable Energy Reviews 2013; 27:362-400, doi:10.1016/j.rser.2013.07.004.

6. Weekes, SM , Tomlin, AS. Data efficient measure-correlate-predict approaches to wind resource assessment for small-scale wind energy. Renewable Energy 2014; 63:162-171, doi:10.1016/j.renene.2013.08.033.

7. Rogers, AL, Rogers, JW , Manwell, JF. Comparison of the performance of four measurecorrelate-predict algorithms. Journal of Wind Engineering and Industrial Aerodynamics 2005; 93(3):243-264, doi:10.1016/j.jweia.2004.12.002.

8. Ayotte, KW. Computational modelling for wind energy assessment. Journal of Wind Engineering and Industrial Aerodynamics 2008; 96(10-11):1571-1590, doi:10.1016/j.jweia.2008.02.002.

9. Palma, JMLM, Castro, FA, Ribeiro, LF, Rodrigues, AH , Pinto, AP. Linear and nonlinear models in wind resource assessment and wind turbine micro-siting in complex terrain. Journal of Wind Engineering and Industrial Aerodynamics 2008; 96(12):2308-2326, doi:10.1016/j.jweia.2008.03.012.

10. Bechmann, A, Sørensen, NN, Berg, J, Mann, J , Réthoré, PE. The Bolund Experiment, Part II: Blind Comparison of Microscale Flow Models. Boundary-Layer Meteorol 2011; 141(2):245271, doi:10.1007/s10546-011-9637-x.

11. Petersen, EL , Troen, I. Wind conditions and resource assessment. Wiley Interdisciplinary Reviews: Energy and Environment 2012; 1(2):206-217, doi:10.1002/wene.4.

12. Weekes, SM, Tomlin, AS, Vosper, SB, Skea, AK, Gallani, ML , Standen, JJ. Long-term wind resource assessment for small and medium-scale turbines using operational forecast data and measure-correlate-predict. Renewable Energy 2015; 81:760-769, doi:10.1016/j.renene.2015.03.066.

13. Brown, A, Milton, S, Cullen, M, Golding, B, Mitchell, J , Shelly, A. Unified Modeling and Prediction of Weather and Climate a 25-Year Journey. B Am Meteorol Soc 2012; 93(12):1865-1877, doi:10.1175/Bams-D-12-00018.1.

14. Tang, Y, Lean, HW , Bornemann, J. The benefits of the Met Office variable resolution NWP model for forecasting convection. Meteorological Applications 2013; 20(4):417-426, doi:10.1002/met.1300.

15. Howard, T , Clark, P. Correction and downscaling of NWP wind speed forecasts. Meteorological Applications 2007; 14(2):105-116, doi:10.1002/met.12.

16. Dee, DP, Uppala, SM, Simmons, AJ, Berrisford, P, Poli, P, Kobayashi, S, Andrae, U, Balmaseda, MA, Balsamo, G, Bauer, P, et al. The ERA-Interim reanalysis: configuration and performance of the data assimilation system. Quarterly Journal of the Royal Meteorological Society 2011; 137(656):553-597, doi:10.1002/qj.828.

17. Grant, ALM , Mason, PJ. Observations of boundary-layer structure over complex terrain. Quarterly Journal of the Royal Meteorological Society 1990; 116(491):159-186, doi:10.1002/qj.49711649107.

18. Wood, N, Mason, P. The Pressure force induced by neutral, turbulent flow over hills. Quarterly Journal of the Royal Meteorological Society 1993; 119(514):1233-1267, doi:10.1002/qj.49711951402.

19. Clark, P, Gallani, M, Hollis, D, Thonson, D, Wilson, C. Small-scale Wind Energy - Technical Report, Urban Wind Energy Research Project, Part 2 - Estimating the Wind Energy Resource.Technical Report Carbon Trust 2008. URL http://www.carbontrust.com/media/85174/small-scale-wind-energy-technical-report.pdf. Date of access: 01/09/2015. 
20. Mason, PJ , King, JC. Measurements and predictions of flow and turbulence over an isolated hill of moderate slope. Quarterly Journal of the Royal Meteorological Society 1985; 111(468):617-640, doi:10.1002/qj.49711146818.

21. Price, JD, Vosper, S, Brown, A, Ross, A, Clark, P, Davies, F, Horlacher, V, Claxton, B, McGregor, JR, Hoare, JS, et al. COLPEX: Field and Numerical Studies over a Region of Small Hills. B Am Meteorol Soc 2011; 92(12):1636-1650, doi:10.1175/2011bams3032.1.

22. Woods, JC, Watson, SJ. A new matrix method of predicting long-term wind roses with MCP. Journal of Wind Engineering and Industrial Aerodynamics 1997; 66(2):85-94, doi:10.1016/S0167-6105(97)00009-3.

23. Standen, J, Wilson, C, Vosper, S. Project report: Remodelling the Irish national onshore and offshore wind atlas.Technical Report SEAI 2013. URL

http://seai.ie/Renewables/Wind_Energy/Wind_Maps/2013-Wind-Atlas-Project-Reports/. Date of access: 01/09/2015.

24. Best, MJ, Pryor, M, Clark, DB, Rooney, GG, Essery, RLH, Ménard, CB, Edwards, JM, Hendry, MA, Porson, A, Gedney, N, et al. The Joint UK Land Environment Simulator (JULES), model description - Part 1: Energy and water fluxes. Geosci. Model Dev. 2011; 4(3):677-699, doi:10.5194/gmd-4-677-2011.

25. Mason, PJ. The formation of areally-averaged roughness lengths. Quarterly Journal of the Royal Meteorological Society 1988; 114(480):399-420, doi:10.1002/qj.49711448007. 
Table 1: Domain specifications.

\begin{tabular}{rccccc}
\hline Domain & $\begin{array}{c}\text { Horizontal } \\
\text { Resolution }\end{array}$ & $\begin{array}{c}\text { Number of } \\
\text { Vertical Levels }\end{array}$ & $\begin{array}{c}\text { Model } \\
\text { Top (km) }\end{array}$ & $\begin{array}{c}\text { Start } \\
\text { Time }\end{array}$ & $\begin{array}{c}\text { Forecast Length } \\
\text { (hours) }\end{array}$ \\
\hline Global & $\begin{array}{c}\text { N216 }(\sim 60 \mathrm{~km} \text { at mid- } \\
\text { latitudes })\end{array}$ & 50 & 63 & 00 UTC & 57 \\
Western Europe & $\begin{array}{c}12 \mathrm{~km} \\
\text { UK }\end{array}$ & 38 & 40 & 06 UTC & 51 \\
\hline
\end{tabular}


Table 2: Verification of hourly instantaneous wind speed from the operational and hindcast winds, calculated at 59 locations at heights $10-200 \mathrm{~m}$ available during $2007-2010$.

\begin{tabular}{rcccc}
\hline & $\begin{array}{c}\text { Mean bias } \\
\left(\mathbf{m s}^{-\mathbf{1}}\right)\end{array}$ & $\begin{array}{c}\text { Standard deviation } \\
\text { of bias }\left(\mathbf{m s}^{-\mathbf{1}}\right)\end{array}$ & $\begin{array}{c}\text { Mean Ibias| } \\
\left(\mathbf{m s}^{\mathbf{- 1}}\right)\end{array}$ & $\begin{array}{c}\text { Standard deviation } \\
\text { of Ibiasl }\left(\mathbf{m s}^{-\mathbf{1}}\right)\end{array}$ \\
\hline Operational archive & -0.31 & 1.06 & 0.65 & 0.90 \\
Reruns & -0.43 & 1.07 & 0.68 & 0.93 \\
\hline
\end{tabular}


Table 3: $R^{2}$ values between observed and modelled hourly, monthly and diurnal meaned wind speed over 1 year at Springhill at $30 \mathrm{~m}$ for the different correction methods - s denotes difference from uncorrected is significant at $5 \%$.

\begin{tabular}{rccc}
\hline & $\begin{array}{c}\text { Hourly } \\
\text { matched } \\
\mathbf{R}^{\mathbf{2}}(\boldsymbol{\%})\end{array}$ & $\begin{array}{c}\text { Monthly } \\
\mathbf{R}^{\mathbf{2}}(\boldsymbol{\%})\end{array}$ & $\begin{array}{c}\text { Mean } \\
\text { diurnal } \\
\mathbf{R}^{\mathbf{2}}(\boldsymbol{\%})\end{array}$ \\
\hline $\begin{array}{r}\text { Uncorrected } \\
\text { Roughness corrected (1A) }\end{array}$ & 65.7 & 95.0 & 93.4 \\
Scaled roughness corrected (1As) & 66.3 & 98.1 & $46.6 \mathrm{~s}$ \\
UKV corrected (1B) & 65.7 & 98.1 & 93.3 \\
Height adjusted (1As\&2A) & 67.1 & 98.1 & 93.3 \\
3D linear model adjusted (1As\&2B) & $68.4 \mathrm{~s}$ & 98.8 & 93.7 \\
UKV and linear model adjusted (1B\&2B) & $67.8 \mathrm{~s}$ & 94.9 & 93.7 \\
\hline
\end{tabular}


Table 4: Summary of verification at 92 locations. * Only 86 locations are within UKV domain, with the 6 excluded being low complexity or offshore. The first column (units $\mathrm{ms}^{-1}$ ) are the absolute values, the second column (units \%) are the mean relative values.

\begin{tabular}{|c|c|c|c|c|c|c|c|c|}
\hline & \multicolumn{2}{|c|}{ Mean bias } & \multicolumn{2}{|c|}{$\begin{array}{c}\text { Standard } \\
\text { deviation of bias }\end{array}$} & \multicolumn{2}{|c|}{ Mean IbiasI } & \multicolumn{2}{|c|}{$\begin{array}{c}\text { Standard } \\
\text { deviation of Ibias| }\end{array}$} \\
\hline & $\mathrm{ms}^{-1}$ & $\%$ & $\mathrm{~ms}^{-1}$ & $\%$ & $\mathrm{~ms}^{-1}$ & $\%$ & $\mathrm{~ms}^{-1}$ & $\%$ \\
\hline Reanalysis & 0.68 & 12.8 & 1.01 & 17.8 & 0.91 & 15.6 & 0.81 & 15.4 \\
\hline Uncorrected & -0.35 & -5.0 & 0.97 & 16.3 & 0.66 & 11.1 & 0.80 & 12.9 \\
\hline $\begin{array}{r}\text { Roughness corrected } \\
\text { (1A) }\end{array}$ & -0.16 & -1.5 & 0.76 & 14.3 & 0.54 & 9.4 & 0.56 & 10.8 \\
\hline $\begin{array}{r}\text { Scaled roughness } \\
\text { corrected (1As) }\end{array}$ & -0.16 & -1.5 & 0.76 & 14.3 & 0.54 & 9.4 & 0.56 & 10.8 \\
\hline UKV corrected $(1 B) *$ & 0.06 & 2.5 & 0.72 & 14.5 & 0.52 & 9.3 & 0.51 & 11.3 \\
\hline $\begin{array}{r}\text { Height adjusted } \\
(1 \mathrm{As \& 2A})\end{array}$ & 0.10 & 1.9 & 0.74 & 12.7 & 0.49 & 8.4 & 0.57 & 9.7 \\
\hline $\begin{array}{r}\text { 3D linear model } \\
\text { adjusted (1As\&2B) }\end{array}$ & 0.10 & 2.5 & 0.65 & 12.9 & 0.45 & 8.3 & 0.48 & 10.2 \\
\hline $\begin{array}{r}\text { UKV and linear } \\
\text { adjustment (1B\&2B)* }\end{array}$ & 0.19 & 4.3 & 0.67 & 12.7 & 0.50 & 9.0 & 0.49 & 10.8 \\
\hline
\end{tabular}


Table 5: Verification of the fully corrected results at 92 sites for the different complexity classifications. The first column (units $\mathrm{ms}^{-1}$ ) are the absolute values, the second column (units \%) are the mean relative values.

\begin{tabular}{rccccccccc}
\hline Complexity & \multirow{2}{*}{$\begin{array}{c}\text { Number } \\
\text { of sites }\end{array}$} & \multicolumn{2}{c}{$\begin{array}{c}\text { Mean bias } \\
\mathrm{ms}^{-1}\end{array}$} & \multicolumn{2}{c}{$\begin{array}{c}\text { Standard } \\
\text { deviation of bias } \\
\mathrm{ms}^{-1}\end{array}$} & \multicolumn{2}{c}{$\begin{array}{c}\text { Mean Ibias| } \\
\mathrm{ms}^{-1}\end{array}$} & \multicolumn{2}{c}{$\begin{array}{c}\text { Standard } \\
\text { deviation of |biasl }\end{array}$} \\
\hline Offshore & 11 & -0.02 & -0.1 & 0.23 & 2.5 & 0.15 & 1.7 & 0.17 & $\%$ \\
\hline Near-shore & 5 & 0.00 & 0.4 & 0.45 & 5.9 & 0.38 & 5.7 & 0.23 & 3.2 \\
Low & 17 & 0.12 & 2.3 & 0.30 & 5.4 & 0.24 & 4.2 & 0.22 & 4.0 \\
Medium & 28 & 0.18 & 3.8 & 0.55 & 10.2 & 0.48 & 8.7 & 0.33 & 6.6 \\
ALL SITES & 31 & 0.06 & 3.0 & 0.98 & 20.2 & 0.74 & 14.4 & 0.65 & 14.5 \\
\hline
\end{tabular}


Table 6: Summary of verification of the UKV corrected (1B\&2B) results over 86 sites for the different complexity classifications. The first column (units $\mathrm{ms}^{-1}$ ) are the absolute values, the second column (units \%) are the mean relative values.

\begin{tabular}{|c|c|c|c|c|c|c|c|c|c|}
\hline \multirow{2}{*}{ Complexity } & \multirow{2}{*}{$\begin{array}{l}\text { Number } \\
\text { of sites }\end{array}$} & \multicolumn{2}{|c|}{ Mean bias } & \multicolumn{2}{|c|}{$\begin{array}{c}\text { Standard } \\
\text { deviation of bias }\end{array}$} & \multicolumn{2}{|c|}{ Mean Ibias| } & \multicolumn{2}{|c|}{$\begin{array}{c}\text { Standard deviation } \\
\text { of Ibias| }\end{array}$} \\
\hline & & $\mathrm{ms}^{-1}$ & $\%$ & $\mathrm{~ms}^{-1}$ & $\%$ & $\mathrm{~ms}^{-1}$ & $\%$ & $\mathrm{~ms}^{-1}$ & $\%$ \\
\hline Offshore & 8 & -0.10 & -1.1 & 0.10 & 1.1 & 0.12 & 1.3 & 0.08 & 0.8 \\
\hline Near-shore & 5 & -0.41 & -5.6 & 0.36 & 4.6 & 0.48 & 7.0 & 0.25 & 3.5 \\
\hline Low & 15 & 0.14 & 2.6 & 0.46 & 7.4 & 0.37 & 6.2 & 0.31 & 4.8 \\
\hline Medium & 28 & 0.36 & 7.2 & 0.51 & 9.8 & 0.50 & 9.2 & 0.38 & 8.0 \\
\hline High & 30 & 0.27 & 6.2 & 0.93 & 18.0 & 0.72 & 13.8 & 0.64 & 13.2 \\
\hline ALL SITES & 86 & 0.19 & 4.3 & 0.67 & 12.7 & 0.50 & 9.0 & 0.49 & 9.9 \\
\hline
\end{tabular}

